\title{
Characterization of the VVV Survey RR Lyrae Population across the Southern Galactic Plane
}

\author{
Dante Minniti ${ }^{1,2,3}$, Istvan Dékány ${ }^{4}$, Daniel Majaess ${ }^{5,6}$, Tali Palma ${ }^{1,2}$, Joyce Pullen ${ }^{1,2}$, Marina Rejkuba ${ }^{7,8}$, Javier Alonso-García ${ }^{2,9}$, \\ Marcio Catelan ${ }^{2,10}$, Rodrigo Contreras Ramos ${ }^{2,10}$, Oscar A. Gonzalez ${ }^{11}$, Maren Hempel ${ }^{10}$, Mike Irwin ${ }^{12}$, Philip W. Lucas ${ }^{13}$, \\ Roberto K. Saito ${ }^{14}$, Patricia Tissera ${ }^{1,2}$, Elena Valenti ${ }^{7}$, and Manuela Zoccali ${ }^{2,10}$ \\ ${ }^{1}$ Departamento de Ciencias Físicas, Facultad de Ciencias Exactas, Universidad Andrés Bello, \\ Av. Fernández Concha 700, Las Condes, Santiago, Chile

\begin{abstract}
Deep near-IR images from the VISTA Variables in the Vía Láctea (VVV) Survey were used to search for RR Lyrae stars in the Southern Galactic plane. A sizable sample of 404 RR Lyrae of type ab stars was identified across a thin slice of the fourth Galactic quadrant $\left(295^{\circ}<\ell<350^{\circ},-2^{\circ} .24<b<-1^{\circ} .05\right)$. The sample's distance distribution exhibits a maximum density that occurs at the bulge tangent point, which implies that this primarily Oosterhoff type I population of RRab stars does not trace the bar delineated by their red clump counterparts. The bulge RR Lyrae population does not extend beyond $\ell \sim 340^{\circ}$, and the sample's spatial distribution presents evidence of density enhancements and substructure that warrants further investigation. Indeed, the sample may be employed to evaluate Galactic evolution models, and is particularly lucrative since half of the discovered RR Lyrae are within reach of Gaia astrometric observations.
\end{abstract}

Key words: Galaxy: structure - stars: variables: RR Lyrae - surveys

Supporting material: machine-readable tables

\section{Introduction}

Optical surveys (e.g., OGLE, La Silla Quest, LINEAR, Catalina, and other surveys) have identified thousands of RR Lyrae stars, which are key tracers of old and metal-poor regions of the Galaxy. In particular, (1) RR Lyrae are excellent reddening indicators, as the stars span a narrow range of intrinsic colors; (2) they are primary standard candles that adhere to well-defined period-luminosity relations; and (3) the pulsators are proxies for age and metallicity, as they constitute an old (age $>10 \mathrm{Gyr}$ ) and metal-poor demographic linked to the primordial stellar population of the Milky Way. This class of variable stars was used to characterize various components of the Milky Way, including the halo (Vivas \& Zinn 2006; Helmi 2008; Miceli et al. 2008; Helmi et al. 2011; Drake et al. 2014, etc.), the bulge (Alcock et al. 2000; Kunder et al. 2008; Dékány et al. 2013; Pietrukowicz et al. 2015; Gran et al. 2016, etc.), the Solar neighborhood (Layden 1994, 1998), and the outer thick disk toward the anticenter (Sesar et al. 2013). RR Lyrae could be used as tracers of accreted galaxies, which might have contributed to the formation of the inner stellar halo-bulge transition region, as suggested by hydrodynamical simulations of MW mass-like galaxies (Tissera et al. 2017).

In a pioneering work, Layden (1994) presented a catalog of RR Lyrae within $5.5 \mathrm{kpc}$ of the Sun, and the sample subsequently became a suite of well-studied local calibrators. Yet it is challenging to identify RR Lyrae throughout the complex Galactic plane region beyond the Solar neighborhood and toward the inner disk, where sizable extinction can render the apparent magnitude beyond the faint limit of most optical surveys.

VISTA Variables in Via Láctea (VVV), a deep near-infrared (NIR) survey, was consequently carried out to identify and characterize RR Lyrae stars in some of the most crowded and reddened regions of the Galaxy. The survey sampled $562 \mathrm{sq}$. deg. of the bulge and the southern disk (Minniti et al. 2010; Saito et al. 2012; M. Hempel et al. 2016, in preparation), enabling the discovery of numerous classes of variable stars concurrently with the RR Lyrae variables, such as bright LPVs/Miras, eclipsing binaries, Cepheids, and microlensing events (Catelan et al. 2013; Minniti et al. 2015). RR Lyrae stars are the focus of the present study, which are concentrated on the lower latitude of the southern disk and its substructures.

The present search for new RR Lyrae candidates expands in part upon our previous work. Dékány et al. (2013) studied bulge RR Lyrae by combining optical with NIR photometry, and discovered that the inner bulge RR Lyrae do not display the barred structure traced by red clump giants, but rather trace an inner spheroidal component. Gran et al. (2016) searched for new RR Lyrae in the bulge-halo transition region, and 

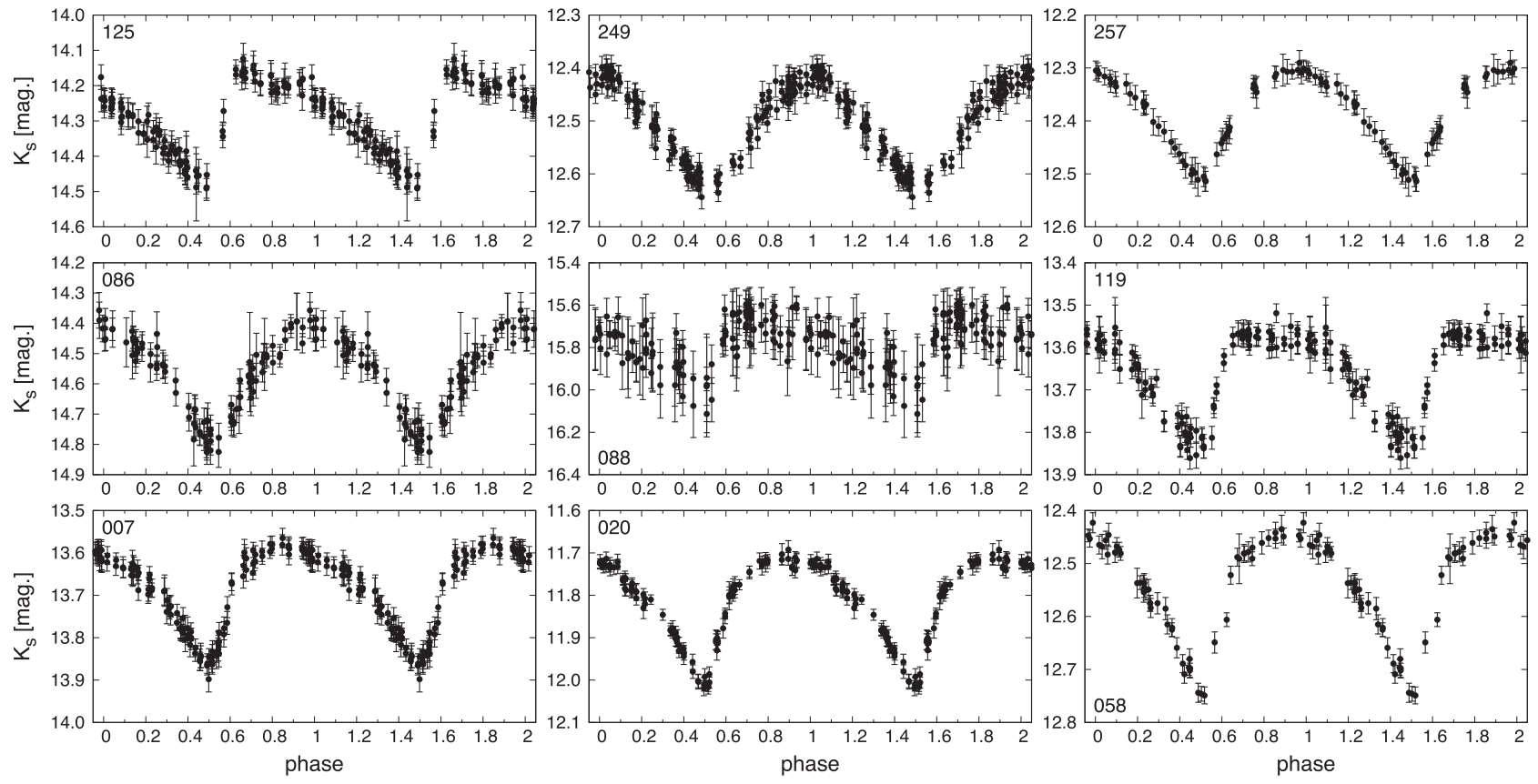

Figure 1. Sample phased light curves for some candidate RR Lyrae type ab. For this work we have concentrated on the search for RRab stars, which are easier to classify because they have asymmetric light curves.

identified 1019 new RR Lyrae type ab variables (RRab), measuring their parameters that included reddening and distance. Those outer bulge RR Lyrae likewise did not adhere to a barred structure. The present work describes the results based on an extended search for RRab stars at low latitudes, whose broader objective is to map the outer bar region and the inner disk. We present the discovery of 404 new RRab stars, which occupy a low latitude slice across the Galactic plane.

This paper is organized as follows. Section 2 describes the selection of the RR Lyrae stars, while Section 3 presents colormagnitude diagrams (CMDs) for our sample. Section 4 discusses the reddening and extinction estimates established, whereas the computed distances are provided in Section 5, which likewise includes a discussion concerning the sample's spatial distribution. In Section 6 we discuss the inferred metallicity estimates, and Section 7 presents a comparison with other RR Lyrae populations. Finally, the conclusions are summarized in Section 8.

\section{The VVV Survey RR Lyrae Selection}

The NIR VVV Survey observations (Minniti et al. 2010; Saito et al. 2012; M. Hempel et al. 2016, in preparation) were acquired with the VIRCAM camera at the VISTA $4.1 \mathrm{~m}$ telescope at ESO Paranal Observatory (Emerson \& Sutherland 2010). In the disk fields typically 70 epochs of observations were acquired in the $K s$-band between the years 2010 and 2015 , in addition to complementary single-epoch observations in the ZYJH bands. The 16 NIR detectors of VIRCAM produce an image of $11 ! 6 \times 11 ! 6$ and a pixel scale of 0 !" $34 /$ pixel. The deep multi-epoch $K_{s}$ band photometry allows us to unveil faint variable sources deep in the disk regions of our Galaxy (Figure 1). A search for RRab stars was made throughout tiles d001 to d038 of the VVV survey's disk field, which is a thin slice through the Galactic plane spanning $295<\ell<350^{\circ}$, and $-2^{\circ} .24<b<-1^{\circ} .05$. The total area covered is nearly $57 \mathrm{sq}$. $\mathrm{deg}$, and the limiting magnitude in the specified tiles spans 17.5 to $18.5 \mathrm{mag}$ in the Ks-band (see the tile positions and maps of Saito et al. 2012).

The search concentrated on RRab stars, because these objects are readily identifiable via their asymmetric light curves, which feature a saw-tooth morphology (Layden 1997). Contrarily, RRc variables feature more sinusoidal light curves, and increased contamination can occur from numerous short period eclipsing binaries that have similar light curve morphology. As a matter of fact, RRc type stars are about 70\% as numerous as RRab type stars in an Oosterhoff I population, and future searches must consider additional properties to select bona fide samples of the former. The amplitudes of NIR RR Lyrae light curves are one-third of those in the optical (e.g., Navarrete et al. 2015).

The procedures outlined by Gran et al. (2015) and AlonsoGarcía et al. (2015) were adopted to identify RRab variables. Approximately $\sim 130,000$ variable star light curves were inspected, and the final selection required that periodic objects fulfilled the following criteria: periods spanning $0.38 \leqslant P \leqslant 1.0$ days, displaying amplitudes of $A_{K_{\mathrm{s}}} \geqslant 0.1 \mathrm{mag}$, and featuring mean magnitudes of $11.5 \leqslant K s \leqslant 16.75$. A limit on the maximum amplitude was not imposed, since the analysis of the observed amplitudes has shown that sometimes they tend to be larger than the real amplitude values due to observational scatter. Moreover, RRab variables possessing periods in excess of 1 day are known to exist; yet such objects display smaller amplitudes in general and are therefore challenging to identify. The final suite of phased light curves (following Dékány et al. 2013) were subsequently visually inspected (e.g., to ensure that they were asymmetric).

The final sample presented here includes 404 objects in total, spread over in 57 sq.deg. Nearly 700 RRab stars were originally identified, and low quality candidates were subsequently culled based on their light curves. Representative light curves of the sample are shown in Figure 1. Table 1 (available in electronic form) provides for all 404 RRab variables IDs, coordinates, observed mean IR magnitudes and 
Table 1

RR Lyrae Sample Photometric Observations

\begin{tabular}{|c|c|c|c|c|c|c|c|c|c|}
\hline VVV-RRL- & R.A. (hms) & Decl. (dms) & $L(\mathrm{deg})$ & $B(\mathrm{deg})$ & $K_{s}$ & $J-K_{s}$ & $H-K_{s}$ & $P$ (days) & Ampl \\
\hline 002 & $11: 51: 57.41$ & $-64: 01: 00.0$ & 296.473 & -1.893 & 14.928 & 0.843 & 0.258 & 0.4567776 & 0.309 \\
\hline 003 & $12: 04: 09.48$ & $-63: 31: 15.2$ & 297.691 & -1.131 & 12.965 & 0.509 & 0.239 & 0.4509296 & 0.349 \\
\hline 004 & $12: 05: 21.20$ & $-63: 37: 34.9$ & 297.841 & -1.211 & 15.633 & 0.880 & 0.266 & 0.4128700 & 0.444 \\
\hline 005 & $12: 05: 25.90$ & $-64: 36: 30.0$ & 298.024 & -2.176 & 14.301 & 0.989 & 0.328 & 0.6404369 & 0.341 \\
\hline 006 & $12: 10: 50.56$ & $-64: 39: 13.5$ & 298.603 & -2.124 & 15.556 & 0.764 & 0.252 & 0.5541016 & 0.213 \\
\hline 007 & $12: 13: 11.06$ & $-64: 14: 55.6$ & 298.791 & -1.685 & 13.675 & 0.968 & 0.302 & 0.6429702 & 0.286 \\
\hline 008 & $12: 16: 10.06$ & $-64: 03: 33.4$ & 299.086 & -1.451 & 14.925 & 0.862 & 0.291 & 0.3751550 & 0.315 \\
\hline 009 & $12: 17: 10.96$ & $-64: 04: 13.5$ & 299.197 & -1.447 & 14.710 & 0.867 & 0.303 & 0.6037623 & 0.338 \\
\hline 011 & $12: 17: 27.52$ & $-64: 46: 56.1$ & 299.321 & -2.149 & 14.406 & 0.647 & 0.203 & 0.6163412 & 0.298 \\
\hline 012 & $12: 18: 42.26$ & $-64: 05: 53.7$ & 299.366 & -1.453 & 13.787 & 0.853 & 0.305 & 0.4762576 & 0.237 \\
\hline
\end{tabular}

(This table is available in its entirety in machine-readable form.)

colors, periods, and amplitudes, as inferred from the $K_{s}$-band light curves. The sample completeness is affected by numerous factors: for example, due to a magnitude-limited survey, faint and low amplitude variables are affected more severely by incompleteness; variables with symmetric light curve shapes may have been discarded to mitigate contamination from eclipsing binaries; Galactic location (varying field density and reddening) may be a factor; RR Lyrae located near the tile edges may possess larger uncertainties, as the images are NIR mosaics; the total number of points per light curve varies between 50 and 130 epochs, therefore affecting the quality of the light curves; and the random temporal sampling of the light curves may introduce period aliases. Note that there are typically 50 independent observations, since the data are often acquired in pairs that are a few minutes apart.

The distribution of observed parameters for the RRab sample are shown in Figure 2 (i.e., locations, magnitudes, periods, and amplitudes). Specifically, the figure conveys the sample's $K_{s}$-band magnitude as a function of the amplitude, Galactic longitude, and period, and a Bailey diagram (i.e., amplitude compared to the period). Our new sample of RR Lyrae in the disk occupies the same regions in these diagrams as the bulge RR Lyrae discovered by Gran et al. (2016). The increased scatter observed arises principally from extreme reddening across the disk fields $\left(0.4<E\left(J-K_{s}\right)<3.5\right)$, while the fields of the outer bulge exhibit $0.0<E\left(J-K_{s}\right)<0.5$ (Gran et al. 2016).

The discrete and smoothed distributions of the new sample of RRab stars as a function of Galactic longitude are shown in Figure 3. The sample spans from R.A. $=205^{\circ}$ to $360^{\circ}$. The distribution expectedly increases toward the Galactic center, given the higher stellar density in the bulge and Galactic center (Valenti et al. 2016). In addition, the observed distribution across the sky is inhomogeneous, displaying maxima and minima.

Figure 4 is a surface density map detailing the positions of the RR Lyrae candidates in Galactic coordinates across $57 \mathrm{sq}$. deg. within 38 tiles. The stars sample a narrow range of Galactic latitude below the plane, from $b=-2.24$ to $b=-1.05$, while Figure 5 conveys the 3D density map of the candidates in equatorial coordinates relative to the $K_{s}$-band magnitudes. The figures highlight the presence of groups of stars that may be associated, and voids are likewise apparent where few stars are located. The spatial distribution is discussed further after the distance estimates are determined (Section 5).

\section{The CMDs}

The NIR CMD was constructed for the RR Lyrae candidates (Figure 6). The objects are significantly brighter than the limiting magnitudes associated with tiles d001 to d038, which range from 17.5 to $18.5 \mathrm{mag}$ in the $K_{s}$-band (Saito et al. 2012). The RR Lyrae are typically fainter than bulge red clump giants, and are bluer than field clump giants. Mean $K_{s}$-band magnitudes were utilized along with single-epoch $J$-band magnitudes to produce $\left(J-K_{s}\right)$, and consequently extra scatter is present due to random phase of J-band observations and periodic color variations. However, the amplitude changes are comparatively small in $\left(J-K_{s}\right)$, and are estimated to be $\Delta$ $\left(J-K_{s}\right)<0.1$ mag (Pietrzynski et al. 2003; Borissova et al. 2009), and the VVV photometric zero points are accurate to 0.02 mag. Thus the principal scatter in color shown in the CMD arises from the extinction spread along the sight-lines to these low latitude RRab stars.

A comparison of Figure 6 with the CMDs of RR Lyrae in other regions of the Galaxy, such as the bulge, strengthens the case that the targets are RR Lyrae located at varying distances along the sight-line through the Galactic disk, with potentially large differences in extinction (see Section 7).

NIR color-magnitude $\left(K_{s}\right.$ versus $\left.\left(H-K_{s}\right)\right)$ and color-color $\left((J-\mathrm{H})\right.$ versus $\left.\left(H-K_{s}\right)\right)$ diagrams were likewise constructed for the RRab candidate sample (Figure 7). The diagrams illustrate the variation in extinction, as demonstrated by the extent of the data along the reddening vector.

\section{Reddenings and Extinctions}

Reddening and extinction values throughout the Galactic plane fields are extreme, and the uncertainties are exacerbated by possible variations in the extinction law (for recent discussions, see Nishiyama et al. 2009; Gonzalez et al. 2012; Chen et al. 2013; Majaess et al. 2016; Nataf et al. 2016). An important advantage of the $V V V$ photometry is that the impact of extinction is significantly reduced in the NIR with respect to the optical (by factors of $\sim 3$ in reddening and $\sim 10$ in extinction).

RR Lyrae stars are excellent reddening indicators because their intrinsic colors are known. They are located in the instability strip, which is defined by a narrow range of temperature and consequently intrinsic NIR colors within $\Delta$ $\left(J-K_{s}\right)=0.10 \mathrm{mag}$ (see Navarrete et al. 2015). Therefore, in principle, their observed colors can be used to estimate individual reddenings along the line of sight. That property has been exploited to characterize the reddening throughout the 

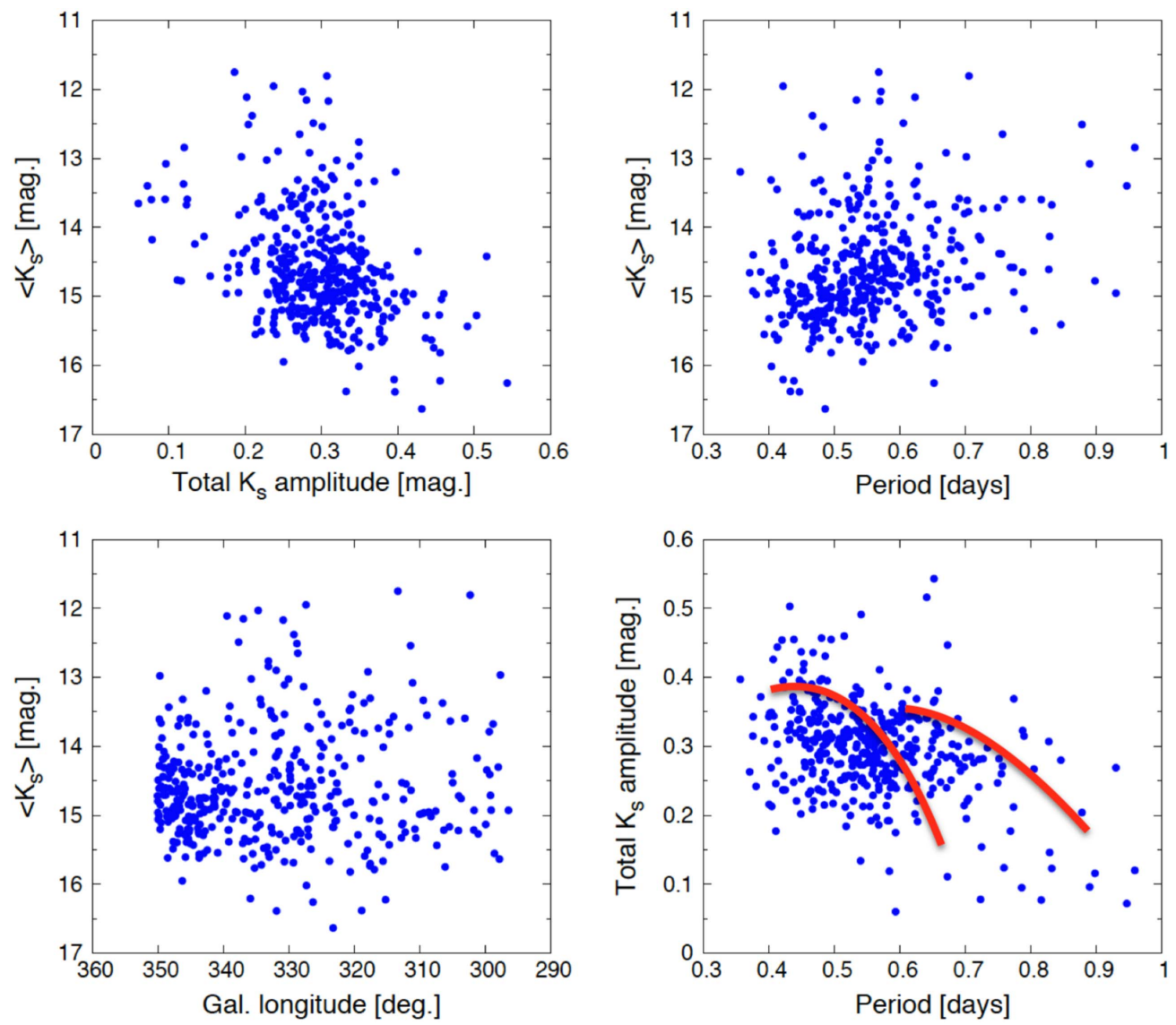

Figure 2. Distribution of magnitudes, periods, and amplitudes of the candidate RR Lyrae. Upper left: magnitudes vs. amplitudes. Upper right: magnitudes vs. periods. Lower left: magnitudes vs. Galactic longitude. Lower right: amplitudes vs. periods (a.k.a. Bailey diagram). The sample RR Lyrae occupy the same regions in these diagrams as the bulge RR Lyrae discovered by Gran et al. (2016). The main near-IR ridge lines for Oosterhoff I and II populations are plotted in red (right and left line, respectively) from Gran et al. (2016).

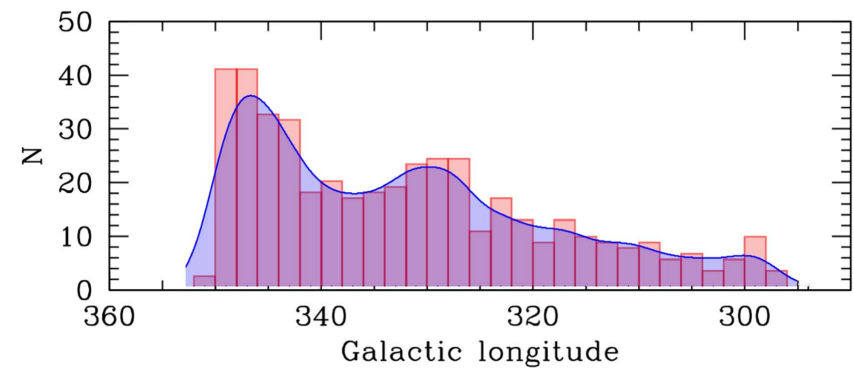

Figure 3. Distribution in Galactic longitude of the newly discovered RR Lyrae type ab stars.

bulge (e.g., Alcock et al. 2000; Popowski et al. 2003) based on optical data. A mean intrinsic (i.e., unreddened) color of $\left(J-K_{s}\right)=0.21 \pm 0.05$ is adopted for all RR Lyrae stars in our sample (see Gran et al. 2016). ${ }^{15}$ Furthermore, as mentioned earlier, the $\left(J-K_{s}\right)$ color range throughout the phase cycle is small, $\Delta\left(J-K_{s}\right)<0.1 \mathrm{mag}$. Exceptions to the adopted mean parameters may be present for RR Lyrae that are blended with field stars. The impact of blending would be bluer observed colors if the blend is with a foreground disk main-sequence

15 There is no consensus about this mean value (e.g., Klein \& Bloom 2014; Gran et al. 2015; Muraveva et al. 2015; Navarrete et al. 2015), but the scatter in this color is so small that it in no case changes significantly the conclusions of this work. star, or redder observed colors if the blend is with a background red giant. The effect of blending is mitigated, since the VVV images exhibit high resolution ( $\sim 0.9$ arcsec), and consequently blends should be rare because the stellar densities at the relevant magnitudes are not very high, even in the low latitude fields observed here. Moreover, the impact of the Blazko effect is reduced in the NIR, and in particular the mean colors are unaffected. ${ }^{16}$

Figure 7 illustrates the significant variation in reddening, as RR Lyrae possessing intrinsic colors $\left(H-K_{S}\right)=0.05 \pm 0.02$ display observed colors spanning from $\left(H-K_{s}\right) \sim 0.0$ to $\left(H-K_{s}\right) \sim 1.0$, and beyond. Certain outliers may be blended stars. The choice of the reddening law is important, and can impact the computed distances. That uncertainty is apparent when comparing the extinction based on reddening laws of Nishiyama et al. (2009) with Cardelli et al. (1989). The extinction is $A_{K_{s}}=0.528 E\left(J-K_{s}\right)$ for Nishiyama, and $A_{K_{s}}=0.72 E\left(J-K_{s}\right)$ for Cardelli, respectively. There is a systematic effect on the distances, depending on the PL relation and the reddening law adopted. It is straightforward to consider the effect of the reddening curve on the distances. The steeper the adopted extinction curve, the smaller the absolute-toselective extinction ratio, and thus the smaller the absolute extinction for the same measured color excess. Therefore, if

\footnotetext{
${ }^{16}$ The Blazko effect produces varying amplitudes for RRab stars (Blazko 1907), and in the optical the colors are often measured at minimum light where they are more stable (Kunder et al. 2010).
} 


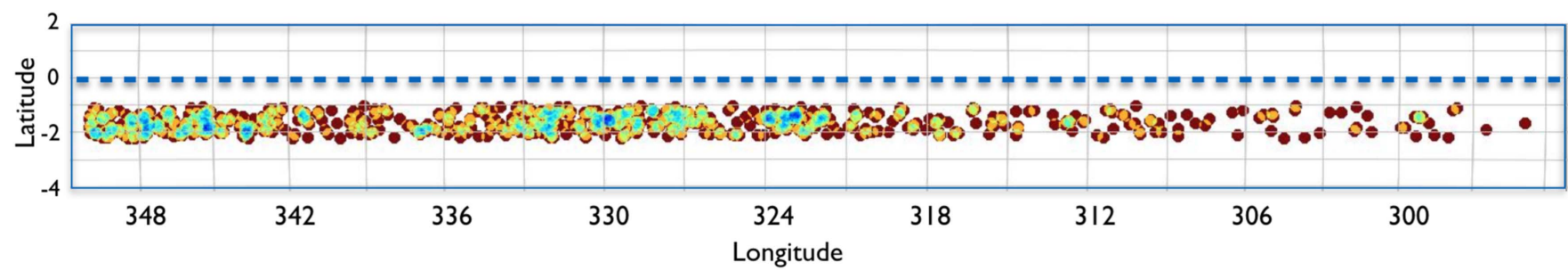

Figure 4. Color surface density map showing the position of the candidate RR Lyrae in Galactic coordinates across 38 tiles at $b=-1.2$, covering 57 sq.deg. in total. The different colors mark the density of overlapping points.

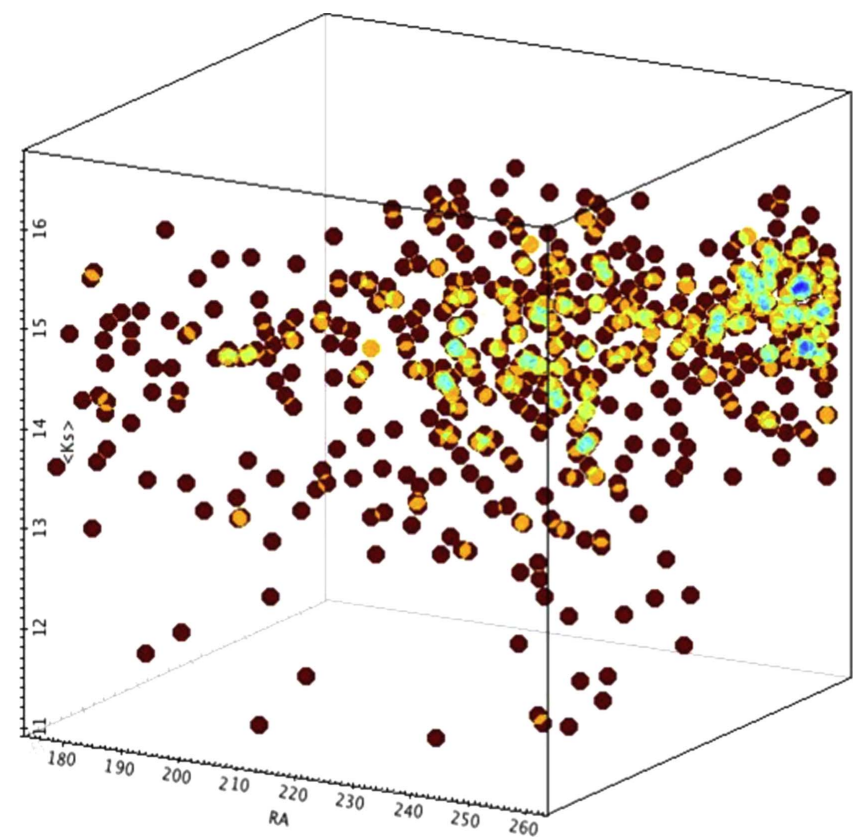

Figure 5. 3D density map showing the position of the candidate RR Lyrae in equatorial coordinates across 38 tiles covering 57 sq.deg. in total.

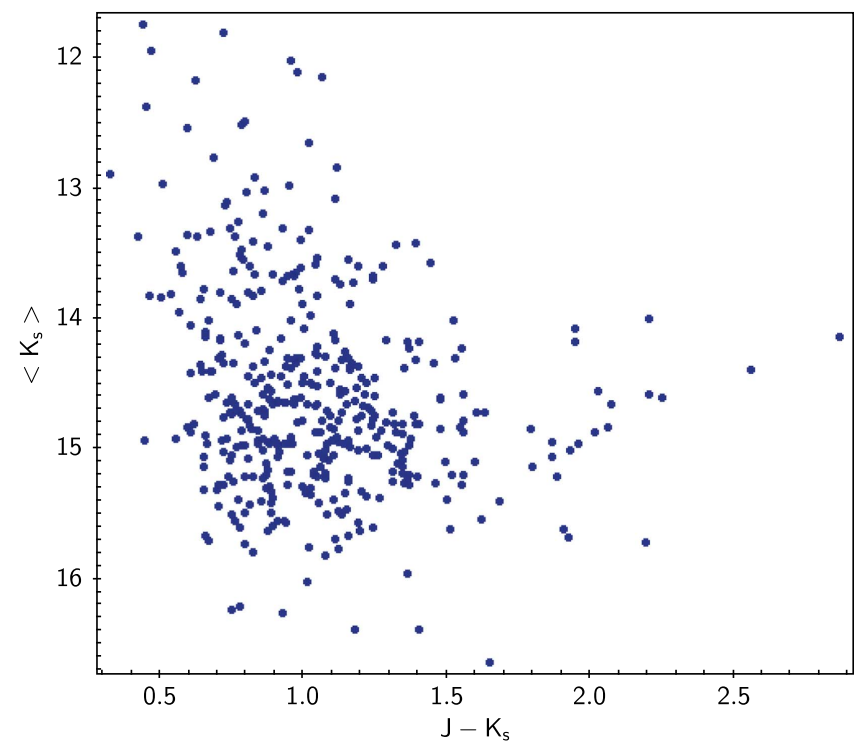

Figure 6. Near-infrared color-magnitude diagram for the RR Lyrae candidates. These objects are much brighter than the limiting magnitudes in the tiles d001 to d038, which range from 17.5 to $18.5 \mathrm{mag}$ in the Ks-band (Saito et al. 2012).

one computes distances adopting a steeper extinction curve, one will get larger distances for the objects for the same color excess values $\left(E\left(J-K_{s}\right)\right.$ in our case). If the assumption about the validity of the Nishiyama extinction curve is incorrect for the present sample, and our objects follow a shallower, more standard extinction curve, then the distances would get systematically shorter.

In sum, the reddening was determined via the following relation: $E\left(J-K_{s}\right)=\left(J-K_{s}\right)-0.21$, where $0.21 \mathrm{mag}$ is the intrinsic $\left(J-K_{s}\right)$ color for RRab stars. The expression adopted to convert the reddening to the total extinction was $A_{K_{s}}=0.528 E\left(J-K_{s}\right)$, because the reddening vector defined by the RRab distribution in the color-color diagram (Figure 7) matches the Nishiyama (2009) result, namely $E(J-H) / E$ $\left(H-K_{s}\right)=2.05$. The sample exhibits a reddening baseline ranging from approximately $E\left(J-K_{s}\right)=0.4$ to 3.0. The equivalent extinction values in the $K_{s}$-band span $0.2<$ $A_{K_{s}}<1.6 \mathrm{mag}$, and in the $V$-band this implies $1.8<$ $A_{V}<14.6 \mathrm{mag}$. The typical reddening value for the sample is $E\left(J-K_{s}\right)=1.0 \mathrm{mag}$, which is equivalent to $A_{K_{s}}=0.5 \mathrm{mag}$ and $A_{V}=4.8 \mathrm{mag}$.

Knowledge of the extinction values and the slope of the reddening law may be utilized to estimate the expected visual magnitudes for the RR Lyrae variables discovered. The objective is to evaluate the possibility of undertaking followup observations for the RR Lyrae sample in the optical, and specifically, if the objects will be surveyed by Gaia. The Gaia mission will revolutionize the study of Galactic structure in general, and of RR Lyrae as distance indicators in particular (e.g., Wilkinson et al. 2005; Bailer-Jones 2009; Eyer et al. 2013; Clementini et al. 2016).

A mock optical-infrared $V$ versus $\left(V-K_{s}\right)$ CMD (Figure 8) was constructed as follows. Mean colors for unreddened Galactic RR Lyrae $(E(B-V) \sim 0.015)$ were adopted from Muraveva et al. (2015). The intrinsic $\left(V-K_{s}\right)$ color for RRab is $M_{V}-M_{K_{s}}=1.006$, and therefore the $K_{s}$-band magnitude corrected for extinction is $K_{s 0}=K_{s}-A_{K_{s}}=K_{s}-0.528$ $E\left(J-K_{S}\right)$ (for the Nishiyama et al. 2009 reddening law). The visual magnitudes were subsequently estimated via $V_{0}=$ $K_{s}-0.528\left(\left(J-K_{s}\right)-0.21\right)+1.0$, where $\left(J-K_{s}\right)_{0}=0.21$ is assumed to be the color of an unreddened RR Lyrae star. The visual magnitude follows from the intrinsic color, extinction, and distance. We indicate the estimated limits for Gaia astrometric and spectroscopic observations (Wilkinson et al. 2005; Clementini et al. 2016) on the CMD in Figure 8. Numerous RR Lyrae stars (approximately half of our sample) have $V<20 \mathrm{mag}$, and are therefore accessible to Gaia astrometric observations. Moreover, about 10 have visual magnitude within reach of the spectroscopic observations with Gaia. Even though Gaia will provide useful proper motions, no useful $(5 \sigma)$ parallaxes are expected for $V>15$ mag sources that are more than $8 \mathrm{kpc}$ away. 

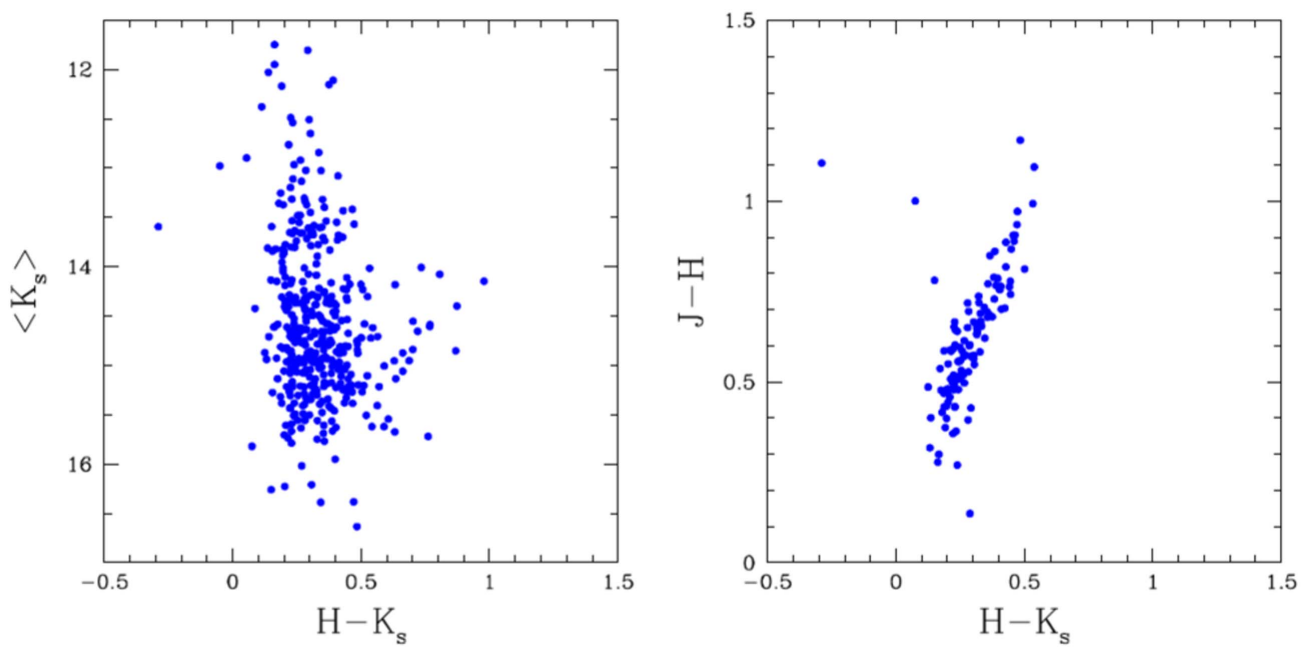

Figure 7. Left panel: $K_{s}$ vs. $\left(H-K_{s}\right)$ near-infrared color-magnitude diagram for the candidate RR Lyrae type ab stars. Right panel: $(J-H)$ vs. $\left(H-K_{s}\right)$ near-infrared color-color diagram for the candidate RRab. The slope of the distribution in this color-color diagram agrees with the reddening vector from Nishiyama et al. (2009).

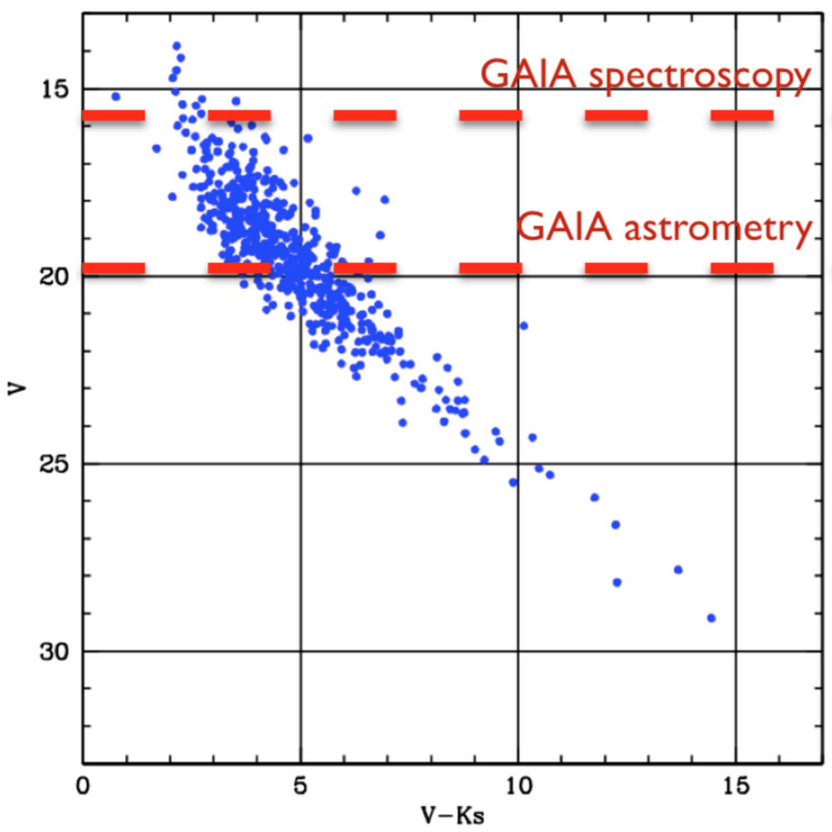

Figure 8. Color-magnitude diagram for the sample RR Lyrae assuming visual magnitudes computed as explained in the text. The limits for Gaia astrometric and spectroscopic observations are shown for comparison.

\section{Distances}

In this section we derive individual distances for our RR Lyrae sample. There are several factors to consider when estimating such distances, beyond the already mentioned issues of high extinction and an uncertain extinction law. Chief among them are the period-luminosity (PL) relations and absolute magnitudes, and their potential dependence on metallicity.

Muraveva et al. (2015) presented recently new near-IR PL and period-luminosity-metallicity $\left(\mathrm{PL}_{K_{\mathrm{s}}} Z\right)$ relations for $\mathrm{RR}$ Lyrae stars, and compared them with the theoretical and empirical relations from the literature (Bono et al. 2003; Catelan et al. 2004; Dall'Ora et al. 2004; Del Principe 2006; Sollima et al. 2006, 2008; Borissova et al. 2009). Based on this, we assume $0.1 \mathrm{mag}$ as an upper limit to the uncertainty for the PL relation when computing the distances. The Muraveva et al.

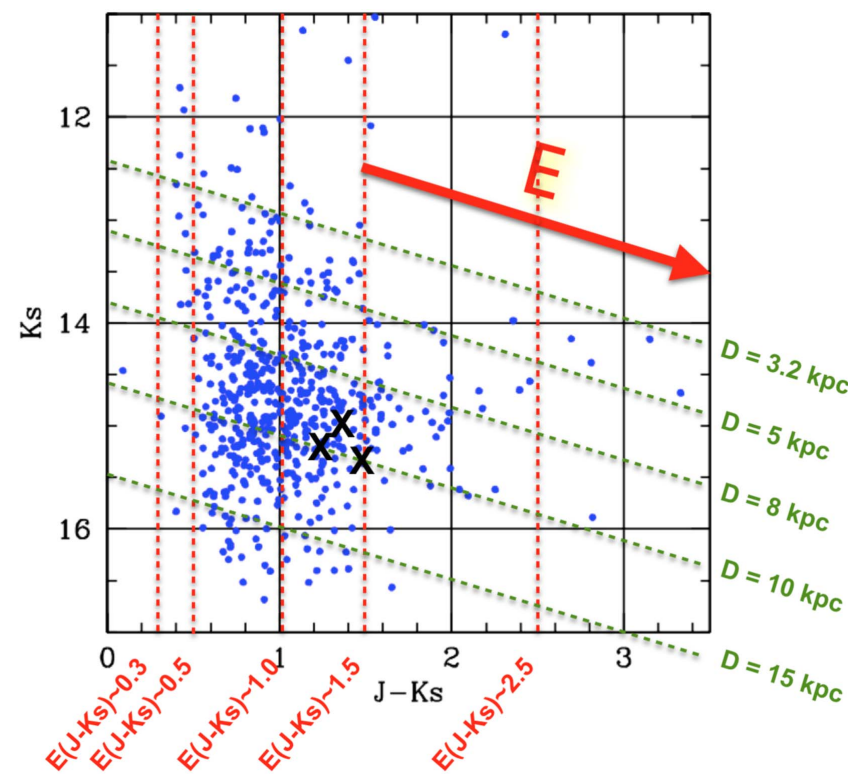

Figure 9. Near-infrared color-magnitude diagram for the candidate RR Lyrae showing lines of constant reddenings and distances. We have assumed the slope of the reddening vector from Nishiyama et al. (2009) as shown. Fiducial reddening and distance lines for the RR Lyrae are marked with dashed red and green lines, as labeled. The X marks the position of two RRab which belong to the globular cluster 2MASS-GC03 (FSR 1735; see Carballo-Bello et al. 2016).

(2015) $\mathrm{PL}_{K_{\mathrm{s}}}$ relation is tied to five RR Lyrae with $H S T$-based parallaxes (Benedict et al. 2011). Distances for our new RRab sample were derived using the PL relation given by Equation (14) of Muraveva et al. (2015)-that is, $M_{K_{s}}=$ $-2.53 \log (P)-0.95$. A period-luminosity-metallicity relation is likewise presented in that work, but the present RR Lyrae sample lacks accurate individual metallicities. While later we use the periods to determine the metallicity distribution, individual metallicities determined in this fashion do not provide improved distance estimates.

A NIR CMD for the RRab sample overlaid with lines of constant reddening and distance is presented in Figure 9. The diagram illustrates the range of distances and extinctions, indicating that our sample becomes incomplete for $D>$ $20 \mathrm{kpc}$, and reddenings and extinctions of $E\left(J-K_{s}\right)>2.5$ and 


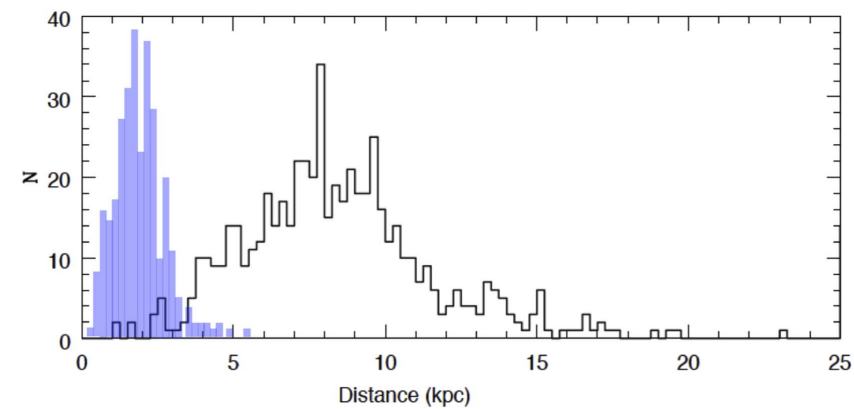

Figure 10. Distance distribution for the RR Lyrae along the line of sight. This is shown with a small bin size $(0.25 \mathrm{kpc})$, in order to illustrate possible groupings along the line of sight. This sample is very complementary to the local sample of Layden (1994), which contains RR Lyrae with $D<5.5 \mathrm{kpc}$ (shown in light blue).

$A_{K_{s}}>1.2 \mathrm{mag}$, respectively. The lines of constant distance were inferred from $P \sim 0.7$ day RRab stars, and targets with different periods will scatter around those representative lines. The CMD excludes 14 stars that lack $J$-band observations, and the reddening vector from Nishiyama et al. (2009) is shown.

There are numerous groups (pairs, triplets) of RRab variables within the discovered sample that lie in close spatial proximity. It is worth following up on these groups as, for example, they may potentially be associated with tidal streams or globular clusters. Three RRab stars appear to belong to the globular cluster 2MASS-GC03 (FSR 1735), located in the VVV tile d031 at R.A. $=16: 52: 11.1, \quad$ Decl. $=-47: 03: 23$, or $l=339.18979$, $b=-1.85318$. The globular cluster provides a valuable anchor to check the computed distances. The positions of the three RRab members of this cluster (V1, V2, and V3 in the notation of Carballo-Bello et al. 2016) are highlighted in Figure 9. CarballoBello et al. (2016) measured a mean cluster metallicity and radial velocity of $[\mathrm{Fe} / \mathrm{H}]=-0.9 \pm 0.2$ and $R V=-78 \pm 12 \mathrm{~km} \mathrm{~s}^{-1}$, respectively. Our estimated mean distance to the RR Lyrae is $D=10.8 \pm 0.4 \mathrm{kpc}$. That distance is in agreement with their position in the cluster CMD (Carballo-Bello et al. 2016). The mean reddening and extinction inferred from the RR Lyrae stars are $E\left(J-K_{s}\right)=1.3 \pm 0.1, A_{K_{s}}=0.69 \mathrm{mag}$, and $A_{V}=6.3$ mag, accordingly. This demonstrates that our distances are reasonable, and justifies the selection of the reddening law, absolute RR Lyrae $M_{K_{\mathrm{s}}}$, and PL relation. In addition, another group of disk RR Lyrae points to the discovery of a new globular cluster in the Milky Way, which we name VVV-GC-05 (FSR1716) and study in detail in a follow-up paper (Minniti et al. 2017).

Our RR Lyrae sample is located in the direction of the thin disk $(-2.24<b<-1.05 \mathrm{deg})$, and complements previous surveys associated with the thick disk, halo, and the wellstudied local sample of Layden (1994). The new RR Lyrae are concentrated toward the Galactic plane, with $0<z<-850$ pc, and exhibit a mean height below the Galactic plane of $-240 \mathrm{pc}$.

The distance distribution for the RRab sample along the line of sight is shown in Figure 10. The density distribution peaks at a distance of $D \sim 8 \mathrm{kpc}$, which is where the RR Lyrae are closest to the Galactic center, and it is also the tangent point with the Galactic bulge. Importantly, the RR Lyrae distribution does not peak at the end of the bulge bar, which is located at a distance of $D=13-14 \mathrm{kpc}$. The result supports claims that the RR Lyrae distribution is not barred (Dékány et al. 2013; Gran

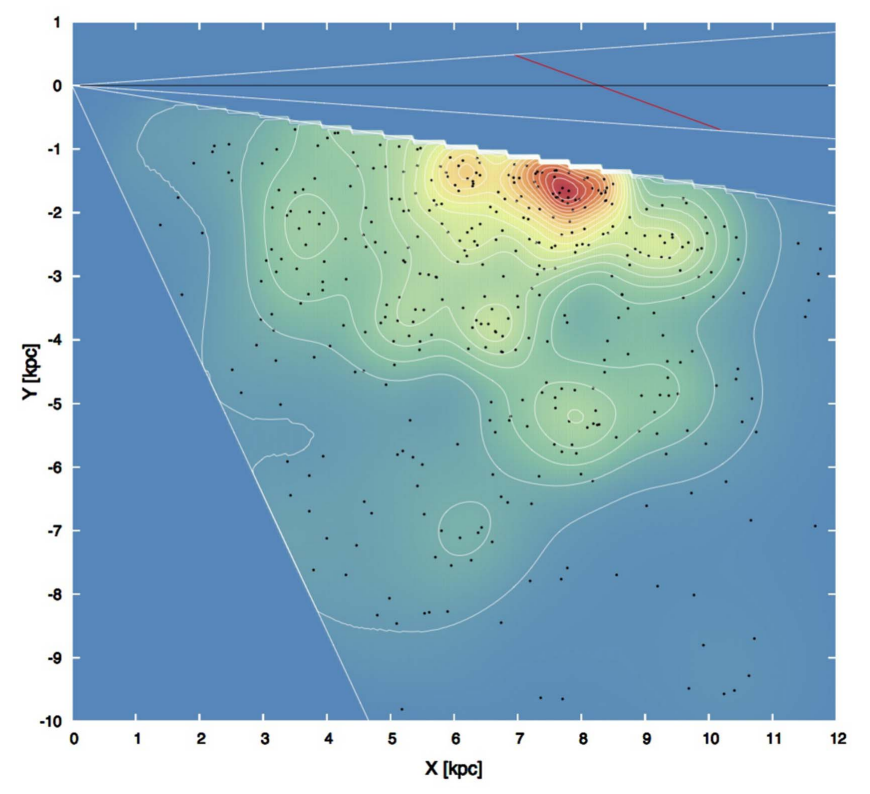

Figure 11. Face-on density map, where the maximum density occurs at the bulge tangent point, as expected if the RRab distribution is not barred but rather spherically symmetric (Dékány et al. 2013; Gran et al. 2016). The spatial substructure of the sample is also apparent. Black dots represent the detected RR Lyr positions. The longitudinal boundaries of the current study and those of Pietrukowicz et al. (2015) are shown with white lines. In addition the position of the long axis of the ellipse fitted by these authors is shown in the red lines (with inclination of $20 \mathrm{deg}$ and Galactic center distance of $8.27 \mathrm{kpc}$, both from their analysis). The Galactic center direction is also shown with a black line.

et al. 2016). An excess number of RR Lyrae lies between 6 and $10 \mathrm{kpc}$, which is the contribution of the bulge demographic.

The distance distribution in Figure 10 appears inhomogeneous, and is potentially revealing true substructure. The distribution is portrayed using a small bin size $(0.25 \mathrm{kpc})$ to illustrate possible groupings along the sight-line. For example, excess RR Lyrae are observed toward tile d026 (centered at $l=332, b=-1.7$ ), and the density enhancement warrants further study. If the observed substructure(s) are not spurious artefacts of small statistics, two separate conclusions may be considered: (1) the distribution may be intrinsically inhomogeneous (e.g., due to halo streams, previously unseen globular clusters or dwarf galaxies, massive disk spiral arms, bulge orbital traps, etc.); (2) the observed distribution is biased by high extinction, as illustrated when comparing the observed spatial distribution with extinction maps (see Figure 4).

Figure 11 conveys a map of the Milky Way together with the distribution of the RRab stars. The figure demonstrates that the peak density is located at the bulge tangent point, as expected if the RRab distribution is not barred, but rather axisymmetric (Dékány et al. 2013; Gran et al. 2016). Furthermore, the bulge RR Lyrae population appears to extend from $\ell \sim 344^{\circ}$, beyond which the excess defined by the bulge RR Lyrae disappears (as seen in Figure 4). Expectedly, Figure 11 likewise shows that the substructures do not trace the spiral arms, and are instead described by a smoother distribution across the Galaxy. Interestingly, certain distinct substructures warrant further investigation. For example, there is a dearth of RR Lyrae in a region of the Galactic plane beyond $10 \mathrm{kpc}$ for longitudes $332<\ell<340^{\circ}$. The absence of remote RRab stars in that region may stem from the presence of a distant thick interstellar cloud producing extreme extinction. In sum, Figure 11 conveys 


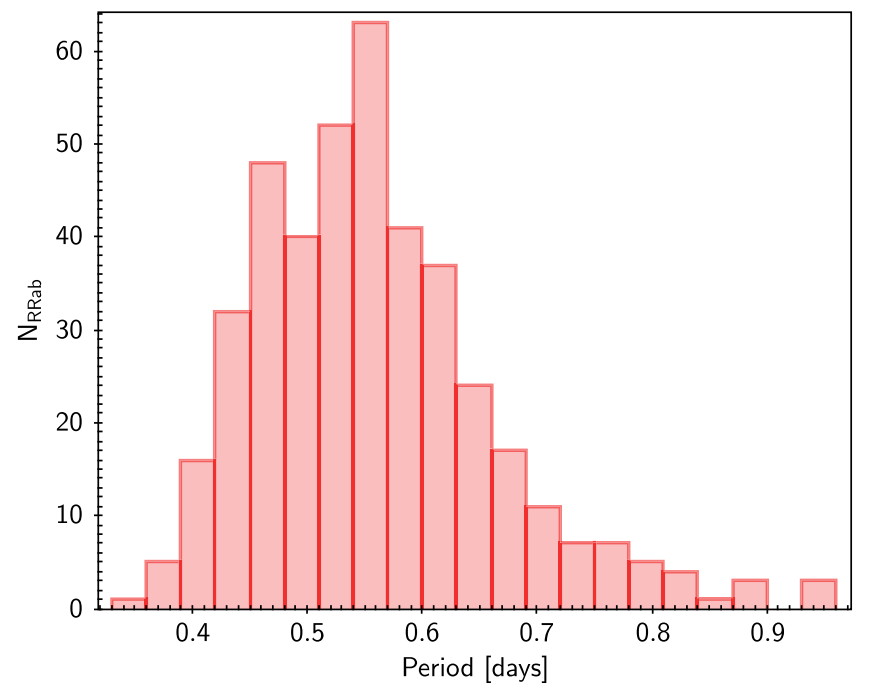

Figure 12. Period distribution for the RR Lyrae sample. The mean of the distribution corresponds to an Oosterhoff type I population (see text).

density enhancements beyond the bulge, which may belong to specific Galactic components (i.e., the disk or the halo).

\section{Metallicities}

A plethora of RR Lyrae exists in the halo fields of the Milky Way, and the stars are likewise often present in sizable numbers in Galactic globular clusters. However, not all globular clusters contain RR Lyrae, and the variables are more common in metal-poor clusters. The observed period distribution for the newly discovered sample of RRab stars exhibits a mean period of $P=0.5586$ days (Figure 12), which indicates that the majority of the RRab resemble an Oosterhoff type I population. RR Lyrae are often divided into two main populations designated Oosterhoff type I or type II (Oosterhoff 1939; Kinman 1959; Catelan 2009). The Oosterhoff populations have different period distributions in globular clusters relative to the halo fields, with the Oosterhoff type I populations displaying shorter mean periods $(P<0.6$ days $)$ and higher metallicities $([\mathrm{Fe} / \mathrm{H}]>-1.5 \mathrm{dex})$ than their type II counterparts (Catelan 2009).

Individual metallicities for RRab stars may be estimated from their periods, with reference to the RR Lyrae calibrators of Layden (1994). Feast et al. (2010) and Yang et al. (2010) cite the following relations: $[\mathrm{Fe} / \mathrm{H}]=-5.62 \log P_{\mathrm{ab}}-2.81$, $\sigma=0.42, \quad$ and $[\mathrm{Fe} / \mathrm{H}]=-7.82 \log P_{\mathrm{ab}}-3.43, \sigma=0.45$, respectively. The derived metallicity distribution for the RR Lyrae sample using the Feast et al. (2010) relation yields a mean metallicity of $[\mathrm{Fe} / \mathrm{H}]=-1.3 \pm 0.2 \mathrm{dex}$ (Figure 13). The individual metallicities were estimated using the periods, and are merely estimates (see discussions by Yang et al. 2010, and Feast et al. 2010) in the absence of spectroscopic determinations. The individual metallicities may have a scatter of several dex, yet the shape of the metallicity distribution confirms that the RRab sample constitutes metal-poor objects, which belong predominantly to an Oosterhoff type I population.

Figure 13 shows the metallicity distribution of the present RR Lyrae sample compared with the RR Lyrae population in the outer bulge from Gran et al. (2016). While the peak locations are similar, the present metallicity distribution is broader than that of the outer bulge sample. This figure shows

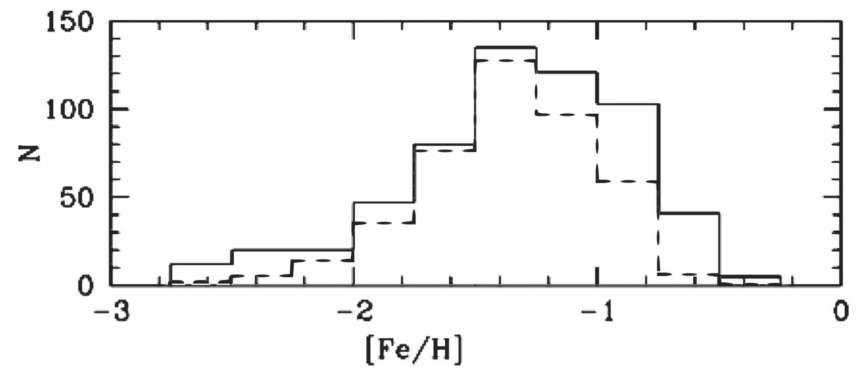

Figure 13. Metallicity distribution for the sample RR Lyrae (black solid line) compared with the sample of Gran et al. (2016) arbitrarily normalized (dashed line). These metallicities were estimated using the periods (Feast et al. 2010; Yang et al. 2010), and should be only considered as rough estimates until spectroscopic determinations become available.

Table 2

Measured Stellar Parameters

\begin{tabular}{lccrr}
\hline \hline VVV-RRL- & $E\left(J-K_{s}\right)$ & $A_{K_{s}}$ & $D(\mathrm{kpc})$ & {$[\mathrm{Fe} / \mathrm{H}]$} \\
\hline 1 & 0.693 & 0.366 & 8.516 & -0.9 \\
2 & 0.359 & 0.190 & 3.716 & -0.9 \\
3 & 0.730 & 0.730 & 11.095 & -0.6 \\
4 & 0.839 & 0.443 & 7.306 & -1.7 \\
5 & 0.614 & 0.324 & 12.783 & -1.4 \\
6 & 0.818 & 0.432 & 5.515 & -1.7 \\
7 & 0.712 & 0.376 & 7.662 & -0.4 \\
8 & 0.717 & 0.378 & 8.819 & -1.6 \\
9 & 0.497 & 0.262 & 8.173 & -1.6 \\
10 & 0.703 & 0.371 & 5.130 & -1.0 \\
\hline
\end{tabular}

(This table is available in its entirety in machine-readable form.)

also that there is a lack of very metal-rich RRab (having up to Solar metallicity), which are seen in the local populations (Layden 1994), suggesting that our sample is dominated by halo RRabs, containing few disk RRab. We predict that the present relatively metal-poor sample should exhibit high velocity dispersion. This needs to be explored spectroscopically, by measuring accurate radial velocities and metallicities.

\section{Comparison with Other RR Lyrae Populations}

The properties for all targets are listed in Table 2 (available in electronic form), which include the reddenings, distances, and metallicities. The RR Lyrae sample discovered is now compared to several results, namely the inner and outer bulge RRab, globular cluster $\omega$ Cen RRab members, the Layden (1994) local sample, and the halo population.

(1) The inner bulge RRab: Dékány et al. (2013) studied the inner bulge RR Lyrae stars and established accurate distances. They concluded that the RR Lyrae distribution is axisymmetric, and not barred. Pietrukowicz et al. (2015) utilized optical photometry from OGLE and contested that result, since they discovered the population adhered to a barred distribution. However, the spatial density distribution of our sample does not peak near the bar's end $(D \sim 13-14 \mathrm{kpc})$, but the maximum is instead observed at the bulge tangent point near $D \sim 8 \mathrm{kpc}$, as expected from an axisymmetric distribution.

(2) The outer bulge RRab: Gran et al. (2016) showed that the outer bulge RR Lyrae do not trace a bar component, whereas a well-defined bar signature is delineated by red clump giants. They argued that the RR Lyrae in the bulge-halo transition have 
an axisymmetric distribution, which supporting the results of Dékány et al. (2013).

(3) The globular cluster $\omega$ Cen: Navarrete et al. (2015) recently completed an NIR analysis of the RR Lyrae population associated with $\omega$ Cen, the most massive Galactic globular cluster, which is considered to be the remnant nucleus of a disrupted dwarf galaxy. $\omega$ Cen is the prototypical example of an Oosterhoff type II population, while the new RR Lyrae sample appears to be a composite population with a large metallicity spread, but dominated by an Oosterhoff type I population, which features a more metal-rich mean $([\mathrm{Fe} / \mathrm{H}]=-1.3)$. Only $\sim 30 \%$ of the sample are of the Oosterhoff type II class like the globular cluster $\omega$ Cen, assuming an arbitrary division of $[\mathrm{Fe} / \mathrm{H}]=-1.5$ for the separation between the Oosterhoff classes.

(4) The local sample: The local sample is likewise a composite Oosterhoff population, but dominated by the Oosterhoff class I RR Lyrae (Layden 1994). These RR Lyrae are located within $5 \mathrm{kpc}$ from the Sun, and appear to be representative of the population found across the plane, since there are no marked differences with remote RR Lyrae discovered in the present study. The new RR Lyrae exhibit Galactocentric distances spanning $R=1.4-20 \mathrm{kpc}$, which extends and complements the Layden (1994) sample, ranging $R=6-13 \mathrm{kpc}$. The present sample features RR Lyrae at smaller Galactocentric distances, and includes RR Lyrae belonging to the outer bulge population, but ultimately the properties are similar to those of local RR Lyrae.

(5) The halo population: There is considerable research concerning the halo RR Lyrae population, because it is a useful tracer of the halo's substructure (e.g., Vivas \& Zinn 2006; Keller et al. 2008; Miceli et al. 2008; Catelan 2009; Sesar et al. 2013; Baker \& Willman 2015). Certain RRab stars in our sample may belong to a halo field population traversing the Galactic plane during their orbits, and under that scenario they should possess high proper motions. Alternatively, if the RR Lyrae are endemic to the Galactic disk, an implication is that the disk is extremely old ( $>10 \mathrm{Gyr}$ ) and must have been in situ since very early in the Milky Way's formation. Followup by Gaia is important in order to establish the kinematics of the RR Lyrae sample, and potential membership within the halo population. In addition, complementary NIR spectroscopy may provide more accurate individual metallicities, and also radial velocities, in order to infer mean orbital properties

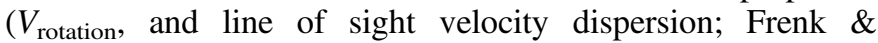
White 1980), which helps clarify disk versus halo versus bulge membership.

\section{Conclusions}

We have discovered 404 RRab candidate variable stars across a 57 sq.deg. region along the southern plane of the Milky Way. NIR photometry from the $V V V$ survey was employed to establish accurate positions, near-IR magnitudes, colors, periods, and amplitudes, as well as to estimate metallicities for the whole sample.

The RRab stars are located at distances spanning from $1<D<30 \mathrm{kpc}$, with the maximum density being at the distance of the Galactic bulge. This indicates that the RRab distribution does not trace the bar delineated by red clump giants, while it is compatible with an axisymmetric distribution (Dékány et al. 2013). Most of the stars have properties indicative of an Oosterhoff type I population. The spatial substructure exhibited in the present sample may be real and warrants further study. For example, three RR Lyrae appear to belong to the Galactic globular cluster 2MASS-GC03 (FSR1735), the other five RRab trace a new globular cluster (VVV-GC05, FSR1716; Minniti et al. 2017), and additional groupings may trace other substructures. While a sizable fraction, close to the Galactic center, belongs to the bulge RR Lyrae population, those further away may belong to a high proper motion halo field population traversing the Galactic plane. These findings may support results from hydrodynamical cosmological simulations where stars formed in satellite galaxies are later on accreted to form part of the inner stellar halo and bulge (Tissera et al. 2017) in Milky Way mass-like galaxies. Alternatively, the RR Lyrae may belong to the Galactic disk, which would imply the disk is old (>10 Gyr) and must have been in place since very early in the Galaxy's formation. Additional observations are necessary to select a scenario, and specifically, proper motions are particularly desirable. The RR Lyrae discovered here are also prime targets for near-IR spectroscopic observations, namely to measure chemical compositions and radial velocities. Lastly, our brightest cataloged RR Lyrae constitute a prime target list for the Gaia mission.

We gratefully acknowledge the use of data from the ESO Public Survey program ID 179.B-2002 taken with the VISTA telescope, and data products from the Cambridge Astronomical Survey Unit (CASU). Support for the authors is provided by the BASAL Center for Astrophysics and Associated Technologies (CATA) through grant PFB-06, and the Ministry for the Economy, Development, and Tourism, Programa Iniciativa Cientifica Milenio, through grant IC120009, awarded to the Millennium Institute of Astrophysics (MAS). D.M., J.A.G., and M.Z. acknowledge support from FONDECYT regular grants no. 1130196, 11150916, and 1150345, respectively. R.K.S. acknowledges support from $\mathrm{CNPq} / \mathrm{Brazil}$ through project310636/2013-2. We are also grateful to the Aspen Center for Physics, where our work was partly supported by National Science Foundation grant PHY-1066293, and by a grant from the Simons Foundation (D.M. and M.Z.).

\section{References}

Alcock, C., Allsman, R., Alves, D. R., et al. 2000, ApJ, 542, 257 Alonso-García, J., Dékány, I., Catelan, M., et al. 2015, AJ, 149, 99 Bailer-Jones, C. A. L. 2009, in IAU Symp. 254, The Galaxy Disk in Cosmological Context, ed. J. Andersen, J. Bland-Hawthorn, \& B. Nordström (Cambridge: Cambridge Univ. Press), 475

Baker, M., \& Willman, B. 2015, AJ, 150, 160

Benedict, G. F., McArthur, B. E., Feast, M. W., et al. 2011, AJ, 142, 187 Blazko, S. 1907, AN, 175, 325

Bono, G., Caputo, F., Castellani, V., et al. 2003, MNRAS, 344, 1097 Borissova, J., Rejkuba, M., Minniti, D., et al. 2009, A\&A, 502, 505 Carballo-Bello, J., Ramirez Alegría, S., Borissova, J., et al. 2016, MNRAS, 462, 501

Cardelli, J. A., Clayton, G. C., \& Mathis, J. S. 1989, ApJ, 345, 245 Catelan, M. 2009, Ap\&SS, 320, 261

Catelan, M., Minniti, D., Lucas, P. W., et al. 2013, arXiv:1310.1996 Catelan, M., Pritzl, B. J., \& Smith, H. A. 2004, ApJS, 154, 633 Chen, B., Schultheis, M., Jiang, B., et al. 2013, A\&A, 550, A42 Clementini, G., Ripepi, V., Leccia, S., et al. 2016, A\&A, 595, A133 Dall'Ora, M., Strom, J., Bono, G., et al. 2004, ApJ, 610, 269 Dékány, I., Minniti, D., \& Catelan, M. 2013, ApJ, 776, 19

Del Principe, M. 2006, ApJ, 652, 362

Drake, A. J., Graham, J., Djorgovski, S. G., et al. 2014, ApJS, 213, 9 Emerson, J. P., \& Sutherland, W. J. 2010, Proc. SPIE, 7733, 773306 Eyer, L., Holl, B., Pourbaix, D., et al. 2013, CEAB, 37, 115 
Feast, M. W., Abedigamba, O. P., \& Whitelock, P. A. 2010, MNRAS, 408, 76 Frenk, C., \& White, S. D. M. 1980, MNRAS, 193, 295

Gonzalez, O. A., Rejkuba, M., Zoccali, M., et al. 2012, A\&A, 543, A13

Gran, F., Minniti, D., Saito, R. K., et al. 2015, A\&A, 575, A114

Gran, F., Minniti, D., Saito, R. K., et al. 2016, A\&A, 591, A145

Helmi, A. 2008, A\&ARv, 15, 145

Helmi, A., Cooper, A. P., White, S. D. M., et al. 2011, ApJL, 733, 7

Keller, S. C., Murphy, S., Prior, S., et al. 2008, ApJ, 678, 851

Kinman, T. D. 1959, MNRAS, 119, 134

Klein, C. R., \& Bloom, J. S. 2014, arXiv:1404.4870

Kunder, A., Chaboyer, B., \& Layden, A. 2010, AJ, 139, 415

Kunder, A., Popowski, P., Cook, K. H., et al. 2008, AJ, 135, 631

Layden, A. C. 1994, AJ, 108, 1016

Layden, A. C. 1995a, AJ, 110, 2288

Layden, A. C. 1995b, AJ, 110, 2312

Layden, A. C. 1997, PASP, 109, 524

Layden, A. C. 1998, AJ, 115, 193

Majaess, D., Turner, D., Dekany, I., Minniti, D., \& Gieren, W. 2016, A\&A, in press (arXiv:1607.08623)

Mateu, C., Vivas, A. K., Downes, J. J., et al. 2012, MNRAS, 427, 3374

Miceli, A., Rest, A., Stubbs, C. W., et al. 2008, ApJ, 678, 865

Minniti, D., Contreras Ramos, R., Alonso-García, J., et al. 2015, ApJL, 810, 20
Minniti, D., Lucas, P. W., Emerson, J. P., et al. 2010, NewA, 15, 433

Minniti, D., Palma, T., Dékány, I., et al. 2017, ApJL, in press

Muraveva, T., Palmer, M., Clementini, G., et al. 2015, ApJ, 807, 127

Nataf, D. M., Gonzalez, O. A., Casagrande, L., et al. 2016, MNRAS, 456, 2692

Navarrete, C., Contreras Ramos, R., Catelan, M., et al. 2015, A\&A, 577, 99

Nishiyama, S., Tamura, M., Hatano, H., et al. 2009, ApJ, 696, 1407

Oosterhoff, P. T. 1939, Obs, 62, 104

Pietrukowicz, P., Kozłowski, S., Skowron, J., et al. 2015, ApJ, 811, 113

Pietrzynski, G., Gieren, W., \& Udalski, A. 2003, AJ, 125, 2494

Popowski, P., Cook, K., \& Becker, A. C. 2003, AJ, 126, 2910

Saito, R. K., Hempel, M., Minniti, D., et al. 2012, A\&A, 537, 107

Schultheis, M., Chen, B. Q., Jiang, B. W., et al. 2014, A\&A, 566, 120

Sesar, B., Ivezić, Z., Stuart, J. S., et al. 2013, AJ, 146, 21

Sollima, A., Cacciari, C., Arkharov, A. A. H., et al. 2008, MNRAS, 384, 1583

Sollima, A., Cacciari, C., \& Valenti, E. 2006, MNRAS, 372, 1675

Tissera, P., et al. 2017, MNRAS, submitted

Torrealba, G., Catelan, M., Drake, A. J., et al. 2015, MNRAS, 446, 2251

Valenti, E., Zoccali, M., Gonzalez, O. A., et al. 2016, A\&A, 587, L6

Vivas, A. K., \& Zinn, R. 2006, AJ, 132, 714

Wilkinson, M. I., Vallenari, A., Turon, C., et al. 2005, MNRAS, 359, 1306

Yang, S. C., Sarajedini, A., Holtzman, J. A., et al. 2010, ApJ, 724, 799 